Proceedings of the XXX International School of Semiconducting Compounds, Jaszowiec 2001

\title{
Interlayer Exchange Coupling in Short Period GaMnAs/GaAs Superlattices
}

\author{
W. Szuszkiewicz, E. Dynowska
}

Institute of Physics, Polish Academy of Sciences

Al. Lotników 32/46, 02-668 Warsaw, Poland

B. Hennion, F. Ott

Laboratoire Leon Brillouin, CE Saclay, 91191 Gif-sur-Yvette cedex, France

M. Jounnne, J.F. Morhange

Laboratoire des Milieux Désordonnés et Hétérogènes, UPMC

4, pl. Jussieu, 75252 Paris, France

M. Karlsteen AND J. SAdOWSKI*

Department of Experimental Physics, Chalmers University of Technology

Göteborg, Sweden

The low dimensional structures containing III-V ferromagnetic semiconductors were intensively investigated in the last few years because of the variety of their potential applications. The aim of the present work was to study the interlayer exchange coupling in the short period GaMnAs/GaAs superlattices containing 16 or 8 GaMnAs monolayers. Samples with the magnetic layers corresponding to the mixed crystal composition with $5 \%$ or $6 \%$ of Mn were grown by MBE technique and characterized by both high-resolution X-ray diffraction and Raman scattering methods. Folded acoustic phonons were observed for the first time for this particular quantum system in the Raman scattering spectra. The interlayer exchange coupling in selected superlattices was investigated by both elastic neutron scattering (diffraction) and polarized neutron reflectivity measurements. Presence of the smooth interfaces and the stability of the superlattice period were confirmed by neutron reflectometry data. Ferromagnetic correlation of the magnetization vector in subsequent GaMnAs magnetic layers was demonstrated by both experimental methods.

PACS numbers: $61.12 .-\mathrm{q}, 75.25 .+\mathrm{z}, 78.30$.Fs

*Also: Institute of Physics, Polish Academy of Sciences, Al. Lotników 32/46, 02-668 Warsaw, Poland. 


\section{Introduction}

GaMnAs belongs to the new class of diluted magnetic semiconductors based on the III-V compounds. It has been obtained for the first time only a few years ago [1]. The low dimensional structures containing III-V ferromagnetic semiconductors have been intensively investigated in the last years because of their potential applications in magnetic memories, spin valves and spin injection or quantum computing devices. In particular, the superlattices (SLs) with the magnetic GaMnAs layers and non-magnetic AlGaAs or InGaAs spacers are of special interest. The disappearance of ferromagnetic properties of GaMnAs single layers has been observed when the layer thickness decreases below a value equal to $\approx 50 \AA$ [2-5]. This opinion can be also found in the review papers (see e.g. [6]).

Very recently, the ferromagnetic properties of the short period (GaMnAs) ${ }_{m} /$ $(\mathrm{GaAs})_{n}$ SLs (where $8 \leq m \leq 12,4 \leq n \leq 8$ ) containing $7 \%$ of Mn have been found in [7]. These findings demonstrated that a set of very thin GaMnAs layers (with each separate layer thickness well below $50 \AA$ ) can also exhibit ferromagnetic behavior. Results of recent Monte Carlo simulations of the ferromagnetism in GaMnAs/AlAs quantum well (based on the confinement-adapted Ruderman-Kittel-Kasuya-Yoshida (RKKY) indirect exchange interaction) predict for the presence of the ferromagnetism in GaMnAs a layer thickness limit of 9 molecular layers $(\mathrm{ML})$, corresponding to a thickness equal to $\approx 25 \AA$ [8]. Results of these simulations are in contradiction to the opinion given in [2-6] but, on the other side, are in agreement with recent experimental data shown in [7].

The interlayer exchange coupling in structures grown on the basis of GaMnAs has been found and reported in a few papers only. Most of those papers concern the physical properties of trilayer structures. GaMnAs/AlGaAs/GaMnAs trilayer properties were investigated in [9] by the magnetotransport and magnetization measurements. The thickness of the magnetic layers was equal to $300 \AA$. It was shown that carriers present in the non-magnetic layer mediate the coupling between the two ferromagnetic layers which has a ferromagnetic character. Similar results were obtained very recently in $[10,11]$. Analysis of the properties of MnAs/GaMnAs/MnAs trilayers demonstrates the presence of ferromagnetic or antiferromagnetic coupling in dependence of the GaMnAs layer thickness [12-14]. Small ferromagnetic coupling was found for MnAs/GaAs/MnAs trilayer [15, 16]. Very recently the change of the character of the magnetic coupling was observed for such trilayer, influence on the heating on the carrier concentration was suggested as possible explanation of this effect [17]. The only superlattices investigated till now were of GaMnAs/AlAs type $[2,4]$ and the ferromagnetic character of the interlayer coupling has been suggested for this system.

There exists very limited information about the interlayer coupling for GaMnAs/GaAs superlattices. Theoretical analysis predicted the oscillations of the ferromagnetic coupling with the GaAs spacer thickness for this system [18]. Such non-monotonic behavior in the magnetization properties of the short period SLs 
were really found recently for the system under consideration [19]. Very recently, the interlayer exchange coupling in GaMnAs/GaAs SLs with a thick (50 $\AA$ or more) GaAs spacers was a topic of studies with the use of a neutron reflectometry [20]. Thus, the aim of the present work was to study possible interlayer exchange coupling in the short period GaMnAs/GaAs SLs by means of the neutron scattering and neutron reflectometry measurements. Up to our knowledge the first method has never been applied for a system based on III-V diluted magnetic semiconductor.

\section{Experimental details}

Two types of samples (containing 16 or 8 GaMnAs ML) were grown for the purpose above mentioned. Samples with the magnetic layers corresponding to the mixed crystal composition with $4.5 \%$ or $6 \%$ of $\mathrm{Mn}$ have been grown by the low-temperature modification of MBE technique directly on (001) oriented semi-insulating GaAs substrate. Investigated SLs have 150 or 200 periods, the thickness of pure GaAs spacer layer was equal to 4, 5, 6, 8, or 10 MLs. Detailed information related to the GaMnAs/GaAs SLs growth can be found elsewhere [7], an information about the growth of the single layers can be found, e.g., in [21].

All SLs were characterized by the high-resolution X-ray diffraction (HRXRD) method. The rocking curves were measured for $004 \mathrm{X}$-ray reflex. Selected samples with $8 \mathrm{GaMnAs}$ MLs were also characterized by the Raman scattering. Raman spectra were taken at room temperature in a wide spectral range (form 3 to $300 \mathrm{~cm}^{-1}$ ) using Jobin-Yvon U1000 spectrometer equipped with a S20 multiplier and photon counting system.

In order to investigate the interlayer exchange coupling in selected SLs, both elastic neutron scattering (diffraction) and polarized neutron reflectivity measurements have been performed using the cold sources in Orphee reactor at Laboratoire Leon Brillouin. SLs were placed in helium exchange gas, the lowest sample temperature was equal to about $11 \mathrm{~K}$. Part of the neutron measurements was performed in the presence of external magnetic field (up to about $0.15 \mathrm{~T}$ for the scattering experiments and up to a few $\mathrm{T}$ for the reflectometry measurements).

\section{Results and discussion}

High sample quality was demonstrated by different characterization methods in spite of a very large number of period repetitions (equal to 150, 200, or 250 in different SLs). In HRXRD spectra well-defined, narrow SLs satellites were observed for all investigated samples which confirmed the SLs period stability and good quality of the interfaces. All measured X-ray diffraction spectra were very well reproduced by the results of numerical simulations, which enable us to determine the values of some parameters describing the properties of investigated samples (exact chemical composition, period, etc.). 
The Raman spectra taken for various short-period SLs show in the low-frequency spectral range the sharp, not very intense structures. These structures were identified as due to acoustic phonon (LA) folding. It is the first observation of such folding in GaMnAs/GaAs SLs. It should be stressed that the folding above mentioned is an evidence of well-defined, sharp interfaces in investigated SLs because the optical density of GaAs and GaMnAs containing a few percent of Mn only is not very different. Observed folded acoustic mode frequency depended on the SL period (on the corresponding to this period wave vector) and such dependence was well described by the linear approximation resulting from the known acoustic wave velocity in pure GaAs.

Apart from the GaAs optical phonon related lines some additional structures were found in the high frequency part of the Raman spectra. These structures resulted from the presence of arsenic in low-temperature (LT) GaAs. This arsenic was introduced into the samples in the technology process. The Raman scattering spectrum for a single, 0.5 micrometer thick LT-GaAs layer was also measured for the comparison. Similar As-related structures were observed in this spectrum which confirms our interpretation given above. Influence of the access of the As in LT-GaAs on the Raman spectrum layers has been analyzed recently in [22, 23] where more detailed discussion of this problem can be found. An example of the Raman spectrum obtained for one of our samples is shown in Fig. 1.

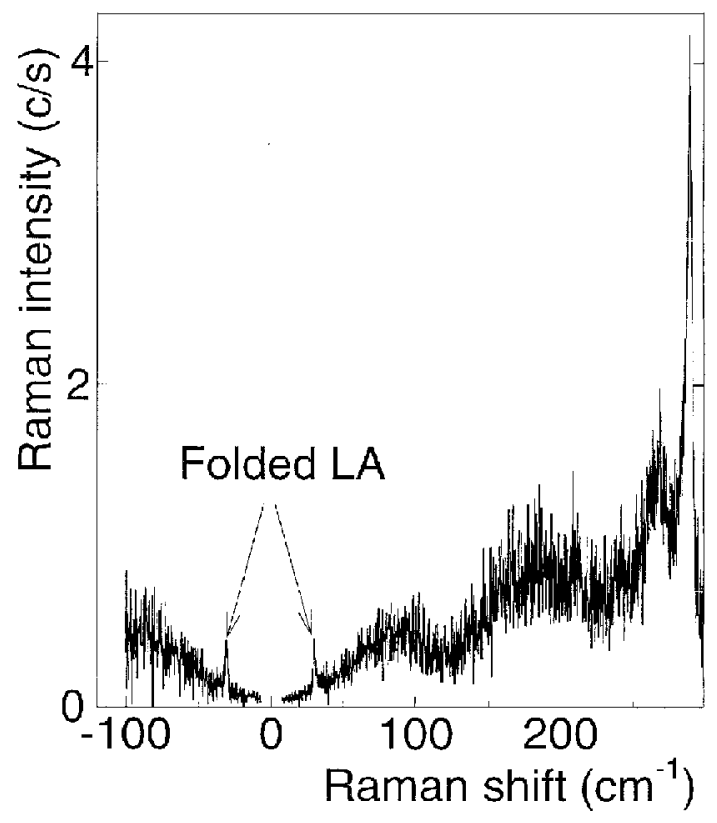

Fig. 1. Raman scattering spectrum taken at room temperature for GaMnAs/GaAs superlattice with 8 magnetic and 8 non-magnetic MLs (details in the text). 


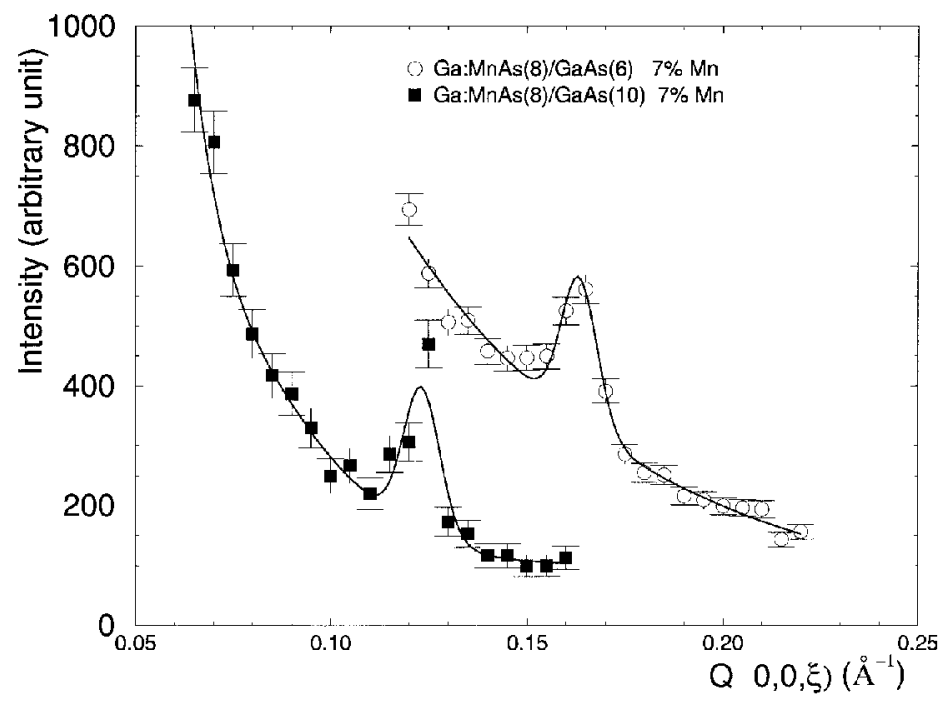

Fig. 2. Small angle neutron scattering spectra corresponding to two SLs with different periods taken at $T=11 \mathrm{~K}\left(k_{i}=1.55 \AA^{-1}\right.$ and $20^{\prime}$ collimations). Observed structure is the satellite line due to the superlattice character of investigated samples. Open circles — data for $(\mathrm{GaMnAs})_{8} /(\mathrm{GaAs})_{6} \mathrm{SL}$, full squares - for (GaMnAs) $8 /(\mathrm{GaAs})_{10} \mathrm{SL}$ (in both cases magnetic slabs contain about $6 \%$ of $\mathrm{Mn}$ ).

Presence of the good quality interfaces and the stable SLs period was also confirmed by neutron reflectometry data which show the smooth wave vector dependence of measured signal. Small angle neutron scattering (diffraction) was measured in the vicinity of the wave vector corresponding to the position of the satellite resulting from the superlattice character of investigated samples. Comparison of the relevant spectra measured for two different samples is shown in Fig. 2. In general, the total intensity of observed satellite is due to both the contribution resulting from the neutron scattering on the chemical (crystal structure) periodicity and the contribution resulting from the magnetic neutron scattering. Analysis of the temperature dependence of this peak intensity enables one to check the hypothesis about the presence of the ferromagnetic interlayer coupling at low temperatures (Fig. 3). As one can see, this intensity slightly increases with decreasing temperature at temperatures below about $30-35 \mathrm{~K}$. It is an evidence of the coupling above mentioned. Such coupling was found for SLs with 16 magnetic MLs and 5 or $6 \mathrm{GaAs}$ MLs, as well as for a system with 8 magnetic MLs only and $6 \mathrm{GaAs}$ MLs. An application of the external magnetic field resulted in the slight increase in the intensity of investigated satellite. An increase in this intensity equal to about $10 \%$ was observed at the magnetic field close to $0.15 \mathrm{~T}$. It means that only a partial magnetic correlation of the magnetization vector exists in the subsequent GaMnAs magnetic layers and the saturation effect could be obtained by the application of the strong enough external magnetic field. 


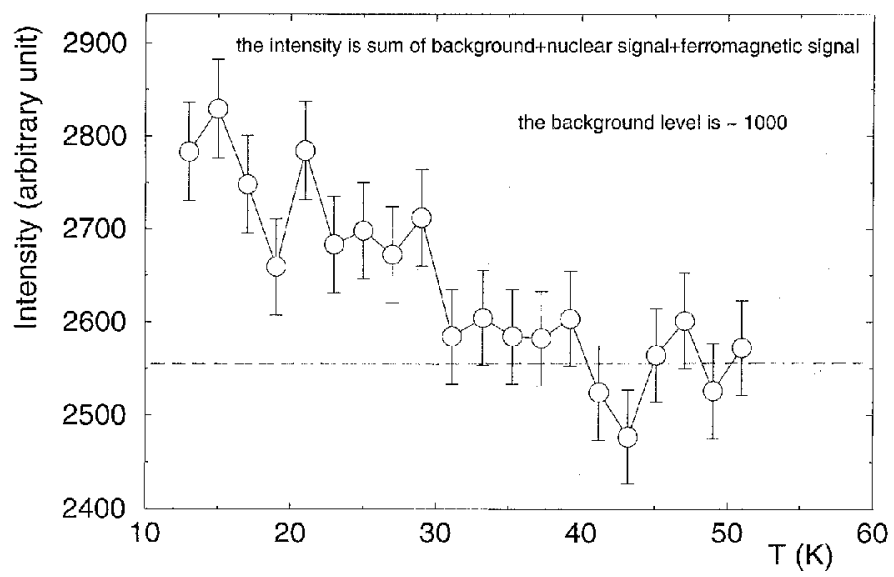

Fig. 3. Intensity of the sattelite peak for (GaMnAs) ${ }_{10} /(\mathrm{GaAs})_{6}$ SL containing $6 \%$ of Mn versus temperature. An increase in the neutron scattering intensity at temperatures below about $35 \mathrm{~K}$ is due to the presence of ferromagnetic correlations between magnetic slabs.

The presence of interlayer magnetic coupling and its ferromagnetic character was also confirmed by means of the neutron reflectometry. No trace of possible magnetic satellite due to the contribution of the antiferromagnetic coupling was detected by both experimental neutron methods. At the present stage of our studies it is difficult to verify the findings suggesting the oscillatory character of the coupling as a function of the non-magnetic spacer thickness. More experimental data corresponding to different superlattices are clearly required for such purpose. This work is in progress and possible results will soon appear.

\section{Acknowledgments}

This work was supported in part within European Community program ICA1-CT-2000-70018 (Centre of Excellence CELDIS) and by the grant 2 P03B 15418 from the State Committee for Scientific Research (Poland). Experiments at LLB were supported by the European Commission through the Access to Research Infrastructures Action of the Improving Human Potential Programme (contract no. HPRI-CT-1999-00032).

\section{References}

[1] H. Ohno, A. Shen, F. Matsukura, A. Oiwa, A. Endo, S. Katsumoto, Y. Tye, Appl. Phys. Lett. 69, 363 (1996).

[2] T. Hayashi, M. Tanaka, K. Seto, T. Nishinaga, K. Ando, Appl. Phys. Lett. 71, 1825 (1997). 
[3] T. Hayashi, M. Tanaka, K. Seto, T. Nishinaga, H. Shimada, K. Ando, J. Appl. Phys. 83, 6551 (1998).

[4] T. Hayashi, M. Tanaka, K. Seto, T. Nishinaga, H. Shimada, H. Hayashi, K. Niihara, Physica E 2, 404 (1998).

[5] A. Shen, H. Ohno, F. Matsukura, H.C. Liu, N. Akiba, Y. Sugawara, T. Kuriowa, Y. Ohno, Physica B 249-251, 809 (1998).

[6] M. Tanaka, J. Vac. Sci. Technol. B 16, 2267 (1998).

[7] J. Sadowski, K. Świątek, P. Svedlindh, R. Mathieu, M. Karlsteen, J. Kanski, L. Ilver, submitted for publication.

[8] M.A. Boselli, A. Ghazali, I.C. da Cunha Lima, Phys. Rev. B 62, 8895 (2000).

[9] N. Akiba, F. Matsukura, A. Shen, Y. Ohno, H. Ohno, A. Oiwa, S. Katsumoto, Y. Iye, Appl. Phys. Lett. 73, 2122 (1998).

[10] D. Chiba, N. Akiba, F. Matsukura, Y. Ohno, H. Ohno, Appl. Phys. Lett. 77, 1873 (2000).

[11] N. Akiba, D. Chiba, K. Nakata, F. Matsukura, Y. Ohno, H. Ohno, J. Appl. Phys. 87, $6436(2000)$.

[12] W. Van Roy, H. Akinaga, S. Miyanishi, K. Tanaka, L.H. Kuo, J. Magn. Magn. Mater. 165, 149 (1997).

[13] W. Van Roy, H. Akinaga, S. Miyanishi, A. Asamitsu, Appl. Phys. Lett. 71, 971 (1997).

[14] H. Akinaga, W. Van Roy, S. Miyanishi, K. Tanaka, J. Appl. Phys. 81, 5345 (1997).

[15] M. Tanaka, K. Saito, T. Nishinaga, Appl. Phys. Lett. 74, 64 (1999).

[16] M. Tanaka, K. Saito, M. Goto, T. Nishinaga, J. Magn. Magn. Mater. 198-199, 719 (1999).

[17] K. Takahashi, M. Tanaka, J. Appl. Phys. 87, 6695 (2000).

[18] T. Jungwirth, W.A. Atkinson, B.H. Lee, A.H. MacDonald, Phys. Rev. B 59, 9818 (1999).

[19] J. Sadowski, P. Svedlindh, R. Mathieu, M. Karlsteen, J. Kanski, L. Ilver, unpublished results.

[20] H. Kepa, J. Kutner-Pielaszek, A. Twardowski, C.F. Majkrzak, J. Sadowski, T. Story, T.M. Giebultowicz, accepted for publication in Phys. Rev. B.

[21] J. Sadowski, J.Z. Domagała, J. Bąk-Misiuk, S. Koleśnik, M. Sawicki, K. Świątek, J. Kanski, L. Ilver, V. Ström, J. Vac. Sci. Technol. B 18, 1697 (2000).

[22] P. Puech, M. Toufella, DR. Carles, R. Sirvin, E. Bedel, C. Fontaine, M. Stellmacher, R. Bisaro, J. Nagle, A. Claverie, G. Benasayang, J. Phys., Condens. Matter 12, 2895 (2000).

[23] H. Sano, A. Suda, T. Hatanaka, G. Mizutani, N. Otsuka, J. Appl. Phys. 88, 3948 (2000). 